Ward, H.; Wenz, L.; Steckel, J. C.; Minx, J. C.

\title{
Truncation Error Estimates in Process Life Cycle Assessment Using Input-Output Analysis
}

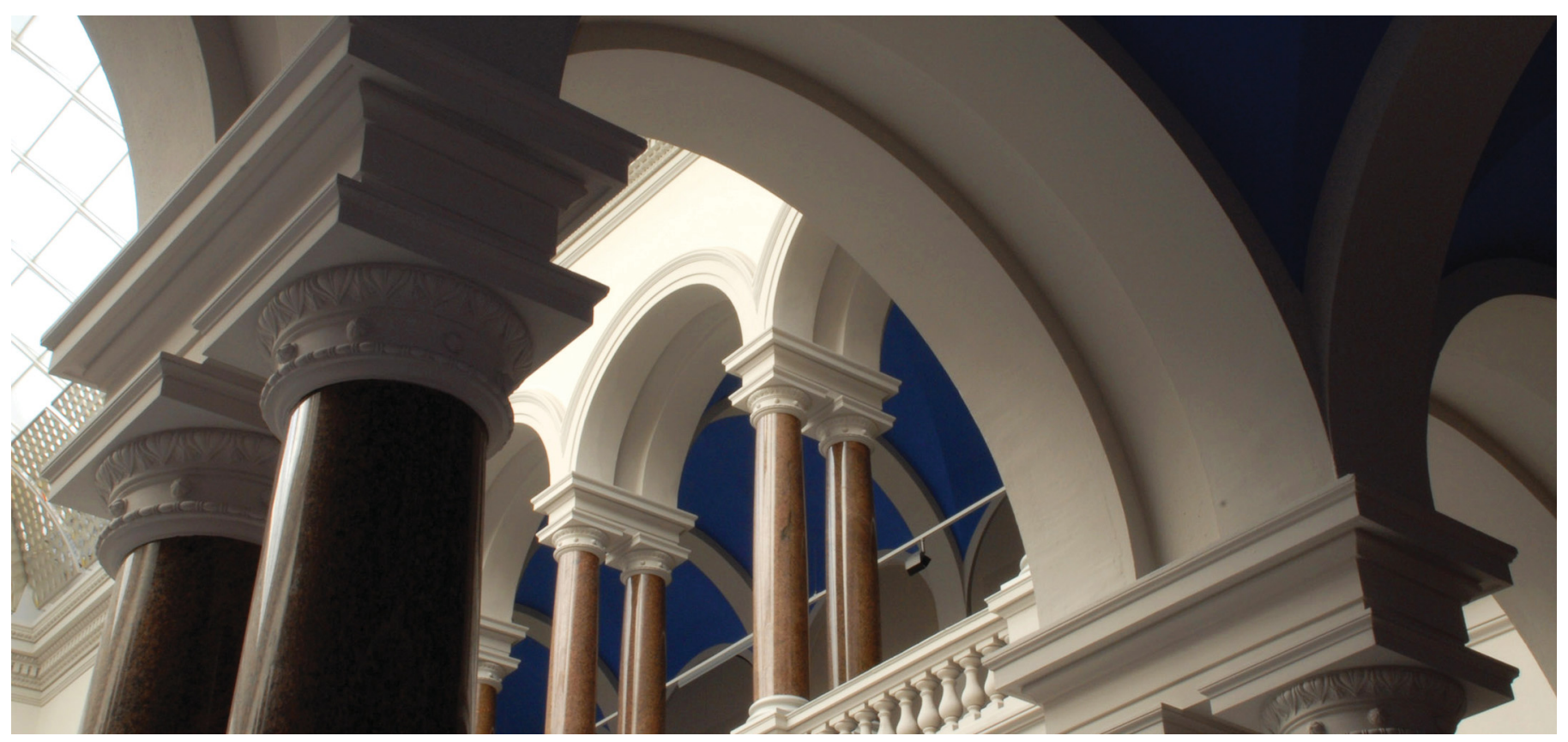

Ward, H., Wenz, L., Steckel, J. C., \& Minx, J. C. (2017). Truncation Error Estimates in Process Life Cycle Assessment Using Input-Output Analysis. Journal of Industrial Ecology, 22(5), 1080-1091. https://doi.org/10.1111/jiec.12655 


\section{Truncation error estimates in process lifecycle 2 assessment using input-output analysis}

3 Hauke Ward*, Leonie Wenz, Jan C Steckel, Jan C Minx

4

5 Keywords: truncation error estimate, process life-cycle assessment, input-output 6 (IO) analysis, system boundary, service sectors

7

\section{Abstract}

9 Process life cycle assessment (PLCA) is widely used to quantify environmental flows associated 10 with the manufacturing of products and other processes. As PLCA always depends on defining a 11 system boundary, its application involves truncation errors. Different methods of estimating 12 truncation errors are proposed in the literature; most of these are based on artificially 13 constructed system complete counterfactuals. In this article we review the literature on 14 truncation errors and their estimates and systematically explore factors that influence truncation 15 error estimates. We classify estimation approaches, together with underlying factors influencing 16 estimation results according to where in the estimation procedure they occur. By contrasting 17 different PLCA truncation error modeling frameworks using the same underlying Input-Output 18 (IO)-dataset and varying cut-off criteria we show that modeling choices can significantly influence 19 estimates for PLCA truncation errors. In addition, we find that differences in IO- and process 20 inventory databases, such as missing service sector activities, can significantly affect estimates of 21 PLCA truncation errors. Our results expose the challenges related to explicit statements on the

\footnotetext{
* Address correspondence to: (postal) Hauke Ward, Mercator Research Institute on Global Commons and Climate Change, Torgauer Straße 12-15, 10829 Berlin, email: ward [at] mcc-berlin.net
} 
22 magnitude of PLCA truncation errors. They also indicate that increasing the strictness of cut-off 23 criteria in PLCA has only limited influence on the resulting truncation errors. We conclude that 24 applying an additional input-output life cycle assessment (IOLCA) or a path exchange hybrid life 25 cycle assessment (HLCA) to identify where significant contributions are located in upstream layers 26 could significantly reduce PLCA truncation errors.

27 


\section{$29<$ heading level $1>$ Introduction}

30 Quantifying environmental impacts of products and processes through the use of a life cycle 31 assessment (LCA) has become standard procedure. Today it is widely applied in research and 32 industry and encouraged by governments and NGOs (Guinée 2011). A range of LCA 33 methodologies are available that utilize different types of data. These are LCA based process-level 34 data (in the following referred to as process LCA or PLCA), LCAs which apply macro-economic 35 input-output data (IOLCA) and methodologies which combine both datasets in so-called hybrid 36 LCA (HLCA). The advantages and disadvantages of each different type of LCA are widely discussed 37 in the literature (see Finnveden et al. (2009) and Rowley et al. (Peters et al. 2010)).

38 PLCA is one of the more frequently applied methodologies. This is possibly because of the high 39 level of detail and availability of the underlying inventory data. These data are regularly 40 distributed together with LCA software tools, making PLCA accessible and generally easy to use 41 (Finnveden et al. 2009). PLCA refers to the iterative bottom-up approach demanded by the ISO 42 norms investigating the environmental interventions related to a product or process by chasing 43 upstream and downstream contributions (British Standards - ISO 14040 2006). Nonetheless, due 44 to practicability, PLCA application requires a system boundary definition. According to the ISO 45 norms, this boundary should be chosen such that the associated process chains are traced until, 46 if possible, all inputs and outputs to the system are flows that have been directly drawn from the 47 environment without human intervention (British Standards - ISO 14044 2006). This ideal 48 condition is difficult to meet in practice, as the number of associated flows potentially grows 49 exponentially and infinitely. Any system boundary definition not only determines the level of 
50 detail that is applied in the analyses, but also the stages, processes, and inputs and outputs that 51 can be deleted if they are not expected to "significantly change the overall conclusion of the 52 study" (British Standards - ISO 14044 2006; British Standards - ISO 14040 2006, p. 8). This means 53 some process flows must be ignored by introducing (explicit) cut-off criteria (Suh et al. 2004), 54 leading to truncation errors. As the complete system is unknown, applying qualitative cut-off 55 criteria that only exclude relevant flows can be challenging (Suh et al. 2004). Put differently, unless 56 100\% of the features of a system are already known, 95\% cannot be calculated (Huang et al. 2009).

57

58 System complete alternative methodologies, based on input-output (IO) frameworks, have been 59 used to construct modeling counterfactuals and estimate the magnitude of truncation errors 60 occurring within a PLCA. In general, although modeling frameworks, as well as resulting estimates 61 vary (Junnila 2006; Lenzen 2000; Norris 2002; Rowley, Lundie, and Peters 2009), studies suggest 62 that truncation errors in PLCA can be significant in size. According to Lenzen (2000) they can be 63 in the order of $50 \%$ of total impacts. Sectors for which relevant impact shares are contained in 64 upstream production stages beyond the second or third layer, are especially prone to truncation 65 errors, as these layers are rarely contained in PLCA studies (Lenzen and Treloar 2003).

66 Approaches for estimating PLCA truncation errors, partially relying on IO data, are based on 67 specific modeling assumptions. They use different methodologies and underlying datasets differ, 68 inter alia, across sectoral resolution (Finnveden et al. 2009; Huang et al. 2009), sectors considered 69 (Majeau-Bettez, Hawkins, and Stromman 2011), and the point in time in which data have arisen 70 or impact categories considered (Finnveden et al. 2009; Suh et al. 2004). It has been shown that 71 depending on the product or process investigated, specific modeling characteristics can lead to 
72 significant differences in results (Huang et al. 2009). Nevertheless, the scientific discourse lacks a 73 systematic investigation into how far different modeling approaches influence PLCA truncation 74 error estimates.

75 In this article we initially provide a comprehensive review of the relevant literature. Based on this, 76 we develop a classification scheme of existing truncation error estimation frameworks and a 77 typology of factors influencing PLCA truncation error estimates. We then investigate in detail the 78 impact of some of the most relevant factors discussed in the literature. We also implement 79 different scenarios, or modeling set-ups. For example we vary cut-off criteria and modeling 80 frameworks, and investigate the influence on PLCA truncation error estimates of service sectors 81 ignored in process inventory databases (Majeau-Bettez, Hawkins, and Stromman 2011). We 82 implement all scenarios using a single IO database for the USA covering more than 400 sectors. 83 For simplicity we only focus on investigating embodied $\mathrm{CO}_{2}$ emissions.

84 Our results show that PLCA truncation error estimates crucially depend on modeling 85 specifications, challenging explicit statements on the magnitudes of truncation errors made in the 86 literature. Note that our results do not examine the quality of PLCA, IOLCA and HLCA techniques 87 as assessment tools. We are primarily concerned with how modeling choices influence truncation 88 error estimates. An investigation of the overall quality of LCA analyses needs a different 89 framework and an understanding of the influence of multiple factors identified in this article. In 90 addition a precise estimate of the overall associated impact is needed, which is difficult to 91 achieve. 


\section{$93<$ heading level $1>$ Truncation errors in PLCA and their estimates}

94 This section formally defines truncation errors in LCAs and reviews the literature on PLCA 95 truncation errors and their estimates. It aims to identify and structure factors that are of 96 relevance for these estimates.

$97<$ heading level $2>$ Truncation error

98 We define a truncation error as the proportion of impact (investigated value) not covered by the 99 system boundaries of the LCA. Truncation errors can occur when flows are knowingly ignored, 100 that is, when their contributions and their upstream flow contributions are - often mistakenly 101 assumed not to affect the overall impact. They can also occur inadvertently when relevant data 102 for the study are (unknowingly) missing and hence flows are disregarded.

103 More formally, we define $M I$ as the measured impact, by which we mean the impact as given by 104 a (P)LCA study of a process or product. TI denotes the corresponding total (unknown) associated 105 impact and EI the estimated total associated impact, as for instance derived from a system 106 complete alternative approach. The related truncation error corresponds to the proportion of the 107 impact that is missing in the assessment as follows:

$108 T E=1-\frac{M I}{T I}$.

109 The corresponding truncation error estimate (TEE) can be expressed as

$$
T E E=1-\frac{M I}{E I}
$$

111 The quality of the estimate consequently depends on how well $T I$ is approximated by $E I$. 112 Nevertheless, a precise number cannot be given, as TI cannot be completely known. 
114 <heading level 2> Factors influencing PLCA truncation errors and their estimates

$116<$ heading level 3> System Boundary

117 The system boundary determines cut-off conditions for flows and associated impacts. It therefore 118 directly influences $M I$. The same holds when the result of a PLCA analysis is approximated within 119 an IO or hybrid framework, see for instance Norris (2002) and Lenzen (2000). A critical point is 120 that the system boundary has to be drawn at the beginning of an LCA study, which is prior to data

121 collection, without knowing the total system and often lacking a scientific basis. This leaves a lot 122 of room for individual interpretation of a "significant contribution" (Suh et al. 2004). ${ }^{\text {ii }}$ Suh et al. 123 (2004) note that an accumulation of small, but disregarded flows could become relevant. Also, 124 small mass or energy content, often used as proxies for the relevance of an impact, do not 125 necessarily correspond to small impacts (Suh et al. 2004).

126

127 <heading level 3> Cut-off criteria

128 No consensus on cut-off methodology across truncation error modeling literature exists; different 129 modeling approaches exist in parallel, indicating that there is no distinct truncation procedure for 130 flows in PLCA, that influences results (see table 1). Considering a specific share of the total 131 footprint or accounting for all flows above a specific anticipated contribution share (British 132 Standard Institute 2011) is difficult in the absence of complete system knowledge (Huang et al. 133 2009). 
134 On the (system-complete) counterfactual side, multiple, different cut-off criteria have been 135 utilized, leading to different results. These cut-off criteria are further explained at the end of this 136 chapter.

137

$138<$ heading level $3>$ Data

139 Missing or incomplete data is another important issue related to the estimation of MI and TI. 140 The choice of data inevitably (and unintentionally) influences truncation errors; only flows 141 included in a database can be considered. For instance some regions are not represented in 142 process databases (PE International 2015), which have also been criticized for ignoring specific 143 service sectors and capital goods (Suh et al. 2004; Junnila 2006). More evidence is given by 144 Majeau-Bettez et al. (2011) who identify explicit sectors contained in an IO dataset which are 145 omitted from process databases, such as government defense, non-defense government and 146 finance services. Additionally, data contained in dissimilar process databases differ from each 147 other (Finnveden et al. 2009; Zhang, Gibbemeyer, and Bakshi 2014). Hence the choice of data has 148 an influence on PLCA results and consequently also on truncation error estimates (as indicated 149 by Huang et al. (2009) by using different IO datasets).

150 On the counterfactual side, all identified approaches are based (at least partially) on IO data, 151 which differ from process inventory databases (see Introduction). IO and PLCA data show 152 differences in the level of sectoral aggregation (Majeau-Bettez, Hawkins, and Stromman 2011; 153 Suh et al. 2004; Junnila 2006; Rowley, Lundie, and Peters 2009; Lenzen 2000) and data contained 154 (Majeau-Bettez, Hawkins, and Stromman 2011). Input-output data uses a monetary accounting 155 system, whereas some PLCA studies account for physical flows (Bruckner et al. 2015). In contrast 
156 to process inventory data, 10 tables typically assume proportionality between monetary and 157 underlying physical flows (Rowley, Lundie, and Peters 2009) and do not consider the gate-to158 grave component (Lenzen 2000). Consequently, they only consider impacts related to production.

159 Many approaches to the estimation of truncation errors are based on 10 datasets for a single 160 region (Rowley, Lundie, and Peters 2009; Lenzen 2000). These yield a higher sectoral resolution 161 than multi-regional IO datasets (Tukker and Dietzenbacher 2013), thus influencing the results (Su 162 et al. 2010). On the downside, they do not consider differences in inter-regional production 163 technologies.

164

165 As a wide range of $\mathrm{IO}$ data exists - constructed in different ways - specific characteristics regarding 166 underlying sectors, regions and impact categories may differ considerably (Tukker and 167 Dietzenbacher 2013). Sectoral aggregation schemes and underlying countries may also vary 168 (Bruckner et al. 2015). Using a specific IO database therefore, has an impact on truncation error 169 estimates (as $M I$ and the approximated $T I$ change), indicated by differences in IOLCA results when 170 using multiple datasets (Ward et al. 2016; Steen-Olsen et al. 2015; Alexeeva-Talebi et al. 2012; 171 Huang et al. 2009).

172 The measured impact is also influenced and potentially falsified by approximating missing data. 173 For example grains have been approximated by wheat due to unavailable data (Peters et al. 2010). 174 In such a case, the resulting truncation error depends on the similarity of the substitute to the 175 missing data. Data can also be supplied by applying matrix inversion techniques to partially 176 compensate for the proportion of impact that has been cut off, or by adding an IO correction term 
177 (holds for HLCA). Both of these influence total measured impacts and can lead to overestimates

178 (Suh and Huppes 2005; Rowley, Lundie, and Peters 2009).

179 <heading level 3> Sectors investigated

180 Truncation error estimates also depend on the sector being investigated, as the related impacts

181 vary in their distribution across different layers (Lenzen and Treloar 2003). For instance, it has

182 been shown that for gas and oil production more than $80 \%$ of the carbon footprint associated 183 with production is contained within the final production step and first upstream layer, whereas

184 in the publishing sector, more than $50 \%$ of the carbon footprint is connected to higher layers 185 (Huang et al. 2009).

186 <heading level 3> Impact investigated

187 A variety of different impacts has been investigated. For instance energy footprints (Treloar 188 1997), emissions footprints (Peters et al. 2010), material footprints (Wiedmann et al. 2013), land 189 use footprints (Bruckner et al. 2015), water footprints (Lenzen et al. 2013) and bad labor, which 190 also considers child labor (Simas et al. 2014) have been assessed. Each impact category has its 191 own characteristic distribution of where relevant impacts are located. Impacts can be difficult or 192 easy to cover by (P)LCA studies depending on this distribution, the supply chain length and 193 structure. A potential truncation error is also dependent on the quality of data coverage. For an 194 impact category, whose impact is insufficiently reported, the occurrence of truncation errors 195 cannot be prevented. 
198 Other sources of truncation errors that have not yet been discussed in the literature might also 199 be relevant. In particular, differences in network properties of 10 data and process databases 200 need to be investigated. Some literature, however, hints of such differences (Mongelli, Suh, and 201 Huppes 2005; Norris 2002). These papers cite differences in the average numbers of network links 202 and differences across other network properties. Typically, for IOLCA, first order upstream flows 203 exceed 300 in number (Norris 2002); it can be expected that this number is much smaller for 204 process inventory databases. A smaller number of direct upstream links implies that more 205 activities are associated with higher process tiers. These characteristics can influence results 206 when investigating (environmental) impacts, using either inventory data or IO data and applying 207 similar cut-off criteria. This is because the second or third layer are rarely contained in PLCA 208 studies (Lenzen and Treloar 2003).

209 Process databases are updated each time a new process is modeled. Consequently, their link 210 density increases over time and converges towards the real density. In contrast, IO data is already 211 system complete and it is likely that this conceptual difference influences corresponding results. 212 To investigate the influence of incomplete link density (and also estimate their real density) the 213 identified power law for self-organized networks (SON) (both, IO tables and complete process 214 inventory database are in principle SON) could be utilized (Laurienti et al. 2011).

215 <heading level 3> Reference Systems

216 In order to estimate the magnitude of a PLCA truncation error, an estimate of $T I$ is needed as well 217 as an estimate of MI. Several approaches have been proposed using system complete data (Suh 
218 et al. 2004). Estimation frameworks have so far (partially) relied on IO data, in which two estimate

219 classes can be identified (see table 1).

220

221 Firstly there are approaches that compare PLCA results with results from system complete 222 alternatives (HLCA or IOLCA) to conclude on computed truncation errors ("between system") 223 (Rowley, Lundie, and Peters 2009). For instance, the IOLCA approach has been used to estimate 224 PLCA truncation errors for energy embodied in basic iron and steel products (estimates are in the 225 order of 50\%) (Lenzen and Dey 2000). By applying two different types of hybrid analyses, a 226 process-based hybrid analysis and an IO-based hybrid analysis, PLCA truncation errors of the life227 cycle energy embodied in passive houses were estimated to be $69 \%$ and $77 \%$, respectively 228 (Crawford and Stephan 2013).

230 Secondly there are approaches that investigate PLCA truncation errors solely within the 231 alternative framework ("within system"). In this way, PLCA application is simulated within an IO 232 framework. The results are then compared to total impacts, which are calculated by IOLCA for the 233 same database (Lenzen 2000; Treloar 1997; Norris 2002).

234 "Within-system" approaches can be sub-classified further. Firstly, a finite layer matrix approach, 235 which we will refer to as "matrix layer approach", was proposed by Lenzen (2000). This uses a 236 power series calculation, where each series element corresponds to a complete layer of upstream 237 flows. For this approach it is crucial to assume that the (applied) truncation of flows in PLCA 238 corresponds to the exclusion of all flows beyond a specific matrix layer $k$ ( $k \in\{0,1,2,3\}$ is often 
239 quoted). Nonetheless, it is questionable whether PLCA flow cut-offs correspond to the practice 240 of matrix layer approaches (Suh et al. 2004). Considering the ISO norms, a judgment on single 241 flows is more appropriate, resulting in flows being cut off in different layers (British Standards 242 ISO 14040 2006; British Standards - ISO 14044 2006).

243 Secondly, path analyses (which we will refer to as "path approaches") have been used to estimate 244 PLCA truncation errors, see for instance Treloar (1997) and Norris (2002). In this way, single 245 entries from 10 tables are used to construct a branching and exponentially growing network of 246 upstream supply flows. In this approach, single flows, that is branches, are traced and 247 investigated. Different variations of this approach exist. For instance, flows can be ranked 248 according to their environmental impact, or a specific number of top contributing flows can be 249 considered (path approach 2.i) (Treloar 1997). Another variant initially ranks all flows and then 250 considers all elements above a specific threshold (path approach 2.iii) ) (Treloar 1997). Norris 251 (2002) considers a specific share of total contribution $(90 \%, 95 \%, 99 \%)$ in each layer to select 252 flows with sufficient contribution (path approach 2.ii)).

253 All these approaches postulate that the total impact is known whereas, in practice, a PLCA 254 applicant has no information on the total impact. Hence, by applying PLCA alone, the entirety of 255 flows cannot be ranked, or the relevant flows located. In this paper we will implement a slightly 256 modified path approach, which in our view better simulates PLCA application considering the ISO 257 norms. This works as follows: if a branch is judged to be significant, its impact is considered and 258 all its direct upstream branches are further investigated; if it is insignificant, it is excluded from 259 the analysis, together with all its upstream flows.

260 An overview of how different estimation frameworks utilize data is given in table 1. 
262 Table 1: Schematic illustration of different approaches to estimate PLCA truncation errors with their corresponding 263 data requirements.

\begin{tabular}{|c|c|c|c|c|c|}
\hline Approach & $\begin{array}{l}\text { Path } \\
\text { approach } 1\end{array}$ & Path approach 2 & $\begin{array}{l}\text { Matrix Layer } \\
\text { approach }\end{array}$ & IOLCA & Hybrid LCA \\
\hline $\begin{array}{l}\text { Within or between } \\
\text { System }\end{array}$ & Within & Within & Within & Between & Between \\
\hline Data used & IO data & IO data & IO data & IO data & $10+$ process data \\
\hline Characteristics & $\begin{array}{l}\text { Upstream } \\
\text { paths are } \\
\text { iteratively } \\
\text { traced }\end{array}$ & $\begin{array}{l}\text { Paths are ranked } \\
\text { according to their } \\
\text { contribution. } \\
\text { Different } \\
\text { possibilities: i) a } \\
\text { specific number } \\
\text { of paths are } \\
\text { considered, ii) a } \\
\text { specific } \\
\text { contribution in } \\
\text { each layer is } \\
\text { considered, } \\
\text { iii) all paths above } \\
\text { a specific } \\
\text { threshold are } \\
\text { considered }\end{array}$ & $\begin{array}{l}\text { Complete } \\
\text { layers are } \\
\text { considered }\end{array}$ & $\begin{array}{l}\text { IOLCA is } \\
\text { performed. } \\
\text { Results are } \\
\text { compared to } \\
\text { PLCA results. }\end{array}$ & $\begin{array}{l}\text { PLOCA and IO are } \\
\text { combined. } \\
\text { Results are } \\
\text { compared to } \\
\text { PLCA results. } \\
\text { Different } \\
\text { approaches exist. }\end{array}$ \\
\hline
\end{tabular}

265 <heading level 3> Varying cut-off criteria within single approaches

266 Threshold schemata differ across path- and matrix-layer approaches, potentially impacting 267 truncation error estimates. For the latter the maximal layer considered varies. For path 268 approaches, absolute and relative thresholds may vary, as well as the specific number of flows 269 that are considered. Generally, other (more) realistic cut-off procedures are conceivable for 270 approximating PLCA within IOLCA. For instance, it is likely that real world LCA applicants add a 271 random component when cutting off flows because of an individual judgment on the relevance 272 of connected impacts (Suh et al. 2004) (please see the Supplementary Information (SI) for a 273 modeling approach). 
$275<$ heading level 3> Truncation error estimation results in the literature

276 Multiple frameworks using different underlying datasets have been applied (see table 1) to

277 estimate PLCA truncation errors (see table 2, which gives examples of different modeling

278 approaches). The findings in the literature suggest a significant variance in magnitudes of 279 truncation error estimates, across modeling frameworks (see table 2) and across sectors (Lenzen 280 2000; Lee and Ma 2013). HLCA frameworks have also been applied; these are separately discussed 281 in the next section. Even though a substantial variation in results can be identified, an examination 282 of how underlying modeling specifications, and the factors identified above, can influence 283 truncation error estimates, using a single reference dataset, is missing. An overview of factors 284 influencing truncation errors and the corresponding estimates is given in figure 1 . Please note 285 that the grey arrows indicate how different datasets are being used to provide an estimate. Our 286 goal is to show that changing modeling specifications can cause a variation in truncation error 287 estimates, using a single underlying dataset.

Table 2 Examples of different approaches used in literature to estimate PLCA truncation errors.

\begin{tabular}{|l|l|l|l|l}
\hline Authors & Lenzen (2000) & Norris (2002) & Treloar (1997) & Rowley et al.(2009) \\
\hline Type of approach & $\begin{array}{l}\text { Matrix Layer } \\
\text { approach for } \\
\text { layers } k=\{0,1,2,3\}\end{array}$ & $\begin{array}{l}\text { Path approach 2.ii); for } \\
\text { each sector, input shares } \\
\text { of }\{90 \%, 95 \%, 99 \%\} \text { are } \\
\text { considered }\end{array}$ & $\begin{array}{l}\text { Path approach 2.iii) with } \\
\text { absolute threshold value } \\
(0.00001 \text { GJ/ \$100 Aus) }\end{array}$ & $\begin{array}{l}\text { HLCA and IOLCA are } \\
\text { compared to PLCA }\end{array}$ \\
\hline IO data used & $\begin{array}{l}\text { Australian } \\
\text { national accounts, } \\
\text { input-output } \\
\text { table, 1994-1995 }\end{array}$ & $\begin{array}{l}\text { U.S. Department of } \\
\text { Commerce's Bureau of } \\
\text { Economic Analysis 1992 }\end{array}$ & $\begin{array}{l}\text { Australian national } \\
\text { accounts, input-output } \\
\text { table, 1986-1987 }\end{array}$ & $\begin{array}{l}\text { Australian national } \\
\text { accounts, input-output } \\
\text { table, 1998-1999 }\end{array}$ \\
\hline
\end{tabular}




\begin{tabular}{l|l|l|l|l}
\hline $\begin{array}{l}\text { Estimated } \\
\text { magnitude }\end{array}$ & $\begin{array}{l}\text { Even when } k=3, \\
\text { truncation error } \\
\text { of up to } 50 \% \text { can } \\
\text { occur }\end{array}$ & $\begin{array}{l}\text { Truncation errors of 23\% } \\
\text { and 35\% result from } \\
\text { shares of } 90 \% \text { and } 95 \%\end{array}$ & $\begin{array}{l}\text { A truncation error of } \\
7.5 \% \text { results (Australian } \\
\text { building sector } \\
\text { considered only) }\end{array}$ & $\begin{array}{l}\text { Truncation error } \\
\text { (evaluated by HLCA) of } \\
3 \% \text { to 55\% for PLCA, } \\
\text { depending on sector and } \\
\text { impact category }\end{array}$ \\
\hline Further insights & $\begin{array}{l}\text { Truncation error } \\
\text { magnitude varies } \\
\text { across sectors }\end{array}$ & $\begin{array}{l}\text { A high degree of system } \\
\text { completeness requires a } \\
\text { huge volume of flows }\end{array}$ & $\begin{array}{l}\text { It is important to consider } \\
\text { energy embodied in } \\
\text { processes more than four } \\
\text { stages upstream }\end{array}$ & $\begin{array}{l}\text { Some IOLCA impacts are } \\
\text { significantly larger than } \\
\text { HLCA and PLCA impacts }\end{array}$ \\
\hline
\end{tabular}

289

290

Sources influencing magnitudes of Systems used for estimating Sources influencing IO based

PLCA TEs

TEs

TEEs

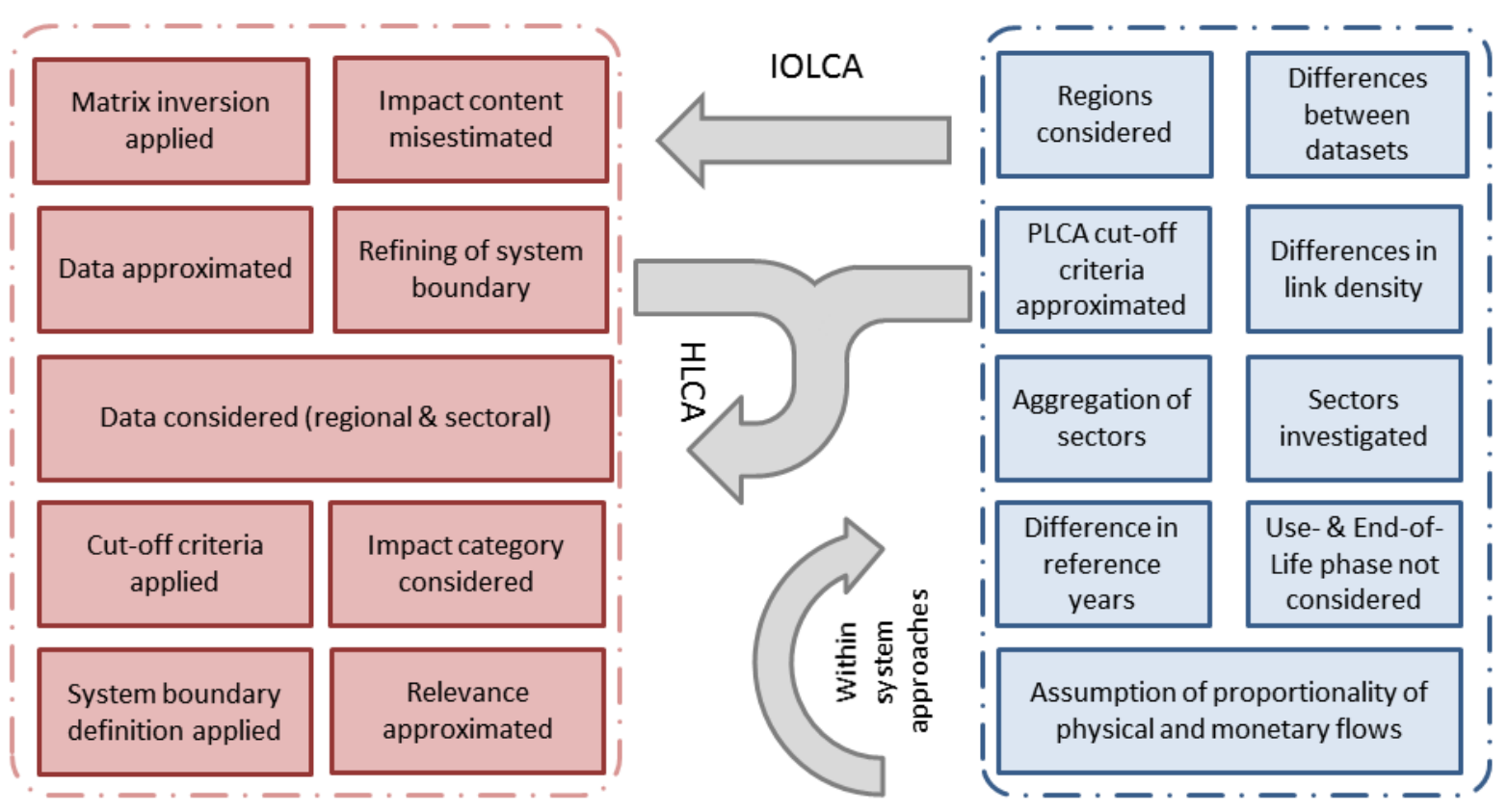

292 Figure 1: Schematic illustration of factors influencing truncation errors (TEs) and truncation error estimates (TEEs).

293 The left side (red) shows factors with direct influence on (unknown) truncation errors in PLCA and PLCA results. The

294 right side (blue) represents factors influencing (system-complete) truncation error estimates. The grey arrows show

295 how estimation approaches utilize different underlying datasets. The origin of an arrow indicates how the total

296 impact has been approximated (either IOLCA or HLCA, which combines PLCA and IOLCA). The head of the arrow 297 indicates how measured impact has been calculated (either using PLCA or an IO based counterfactual).

299 <heading level 2> Excursus Hybrid LCA for estimating truncation errors

300 Both PLCA and IOLCA have short-comings. PLCA analysis misses the impact associated with higher

301 layers (Lenzen and Treloar 2002; Crawford and Stephan 2013) that can significantly change the 
302 conclusion of (comparative) studies (Lenzen and Treloar 2003). System complete IOLCA lacks the 303 extensive detail of process inventory databases, as well a gate-to-grave component (Suh and 304 Huppes 2005; Lenzen 2000). Hence, literature judges the application of HLCA, combining the 305 advantages of both approaches, to be superior (Wiedmann et al. 2011; Suh and Huppes 2005; 306 Lenzen and Crawford 2009; Huang et al. 2009; Crawford and Stephan 2013; Rowley, Lundie, and 307 Peters 2009; Guinée 2011). Different approaches for HLCA exist, inter-alia tiered-HLCA (Suh and 308 Huppes 2005), the path exchange method (Lenzen and Crawford 2009) and the IO-based HLCA 309 (Joshi 1999).

310 It has been shown that, depending on the type of hybrid LCA that is applied to the activity 311 investigated, both IOLCA and PLCA can miss relevant impact shares if HLCA is assumed to be 312 precise (Rowley, Lundie, and Peters 2009; Wiedmann et al. 2011; Crawford and Stephan 2013). It 313 has also been revealed that IOLCA can overestimate specific impacts (Rowley, Lundie, and Peters 314 2009); depending on the environmental indicator and sector considered, and assuming that HLCA 315 results can serve as a reference, estimates for truncation errors for PLCA are in the range of $2 \%$ 316 to 77\% (Rowley, Lundie, and Peters 2009; Wiedmann et al. 2011; Crawford and Stephan 2013). 317 Corresponding estimates for IOLCA are in the range of $-51 \%$ to $96 \%$ (Rowley, Lundie, and Peters 318 2009; Crawford and Stephan 2013).

319 Nevertheless, as one cannot compare HLCA to a better reference system (as it is partially consists 320 of PLCA whose complete impact remains unknown), it is difficult to judge the quality of the 321 estimate. In addition, if two HLCA approaches are applied, as in Wiedmann et al. (2011) and 322 Crawford and Stephan (2013), it is impossible to judge which one is closer to the real unknown 323 impact. 
324 Although it is highly likely that HLCA produces better results, we do not use it for our simulations,

325 as a qualitative reference, to which results can be compared, is lacking. In addition, implementing

326 HLCA within an IO framework, where the total impact is known, would result in an IOLCA.

$327<$ heading level 2> Analysis framework

328 This section describes the framework used to investigate the influence of the most relevant 329 modeling configurations of PLCA truncation error estimates in literature. We implement different 330 estimation frameworks, vary threshold rules and disregard specific service sectors in scenarios.

331 Finally, we contrast their results. When doing so, we do not aim to provide exact estimates; this 332 is impossible as the total impact in the process inventory system remains unknown. Our goal is to 333 assess whether there is relevant influence by modeling specifications. We avoid estimation errors 334 linked to the comparison of two different systems, that is "between system", by implementing 335 scenarios and evaluating truncation error estimates within a single IO system (within system).

$336<$ heading level 3> Data

337 In order to implement the different modeling frameworks we use the single region Open 10 338 database for the US (Applied Sustainability Center, University of Arkansas. Sylvatica 2010), 339 representing the US economy in 2002. The database has a resolution of 430 sectors and also 340 provides data on different greenhouse gas releases. For demonstration purposes, only $\mathrm{CO}_{2}$ 341 emissions are considered in this article. As we use a single region IO table, exports and imports 342 are not accounted for separately. It is assumed that other countries produce commodities with 343 the same sectoral coefficients. 
344 Although IO databases with higher resolution exists, for instance the national Australian 10 with

3451284 sectors (available at (IELab 2017)), we use the US database, as it fits our purposes perfectly.

346 This is because it has been used by Majeau-Bettez et al. (2011), who precisely identified and

347 named the sectors that are excluded from inventory databases. Hence, it allows us to easily

348 exclude such sectors. Taking a different dataset, with varying sectors, would require us to

349 approximate the sectors identified by Majeau-Bettez et al. (2011), potentially introducing 350 additional errors.

$351<$ heading level 3> IO notations

352 Standardized IO data consist of an inter-industry flow matrix $Z \in \mathbb{R}^{m x m}$ and a final demand vector $353 Y \in \mathbb{R}^{m}$. Entries $Z_{u}^{v}$ of $Z$ reflect the total monetary value (in USD) of flows from sector $u$ to sector $354 v$ with $u, v \in M=\{1, \ldots, m\}$; where $m$ denotes the number of all sectors. Analogously, $Y_{u}$ 355 represents the sum of all monetary flows from sector $u$ into final demand.

356 By $O \in \mathbb{R}^{m}$, we denote the total output vector, with entries $O_{u}=\sum_{v} Z_{u}^{v}+Y_{u}$, giving the total 357 output of sector $u . A \in \mathbb{R}^{m x m}$ denotes the technology matrix, consisting of entries $A_{u}^{v}=Z_{u}^{v} / O_{v}$, 358 that describe the amount of each input $u$ (in USD) that is required by sector $v$ in order to produce 359 one unit of output (in USD).

360 The Leontief inverse is calculated as $L=(I-A)^{-1}$, where $I$ denotes the unity matrix. It 361 accounts for all pre-products that have been used at some stage during the production process. 362 Further, $363 \sum_{l=0} A^{l} \rightarrow(I-A)^{-1}$ for $l \rightarrow \infty$ holds, where each term of the power series refers to a 364 complete upstream production tier. 
365 Additionally, we use data on released $\mathrm{CO}_{2}$ emissions. We let $F \in \mathbb{R}^{m}$ denote the vector whose $m$ 366 entries $F^{u}$ denote total emissions released by sector $u$. Dividing $F$ by total sectoral outputs $O$ 367 results in vector $f \in \mathbb{R}^{m}$ whose entries $f^{u}$ reflect $\mathrm{CO}_{2}$ emissions associated with one USD of 368 output of sector $u$.

369

$370<$ heading level 3> 10 - Matrix layer approach

371 When applying the matrix layer approach to estimate truncation errors, it has been assumed

372 that the PLCA application can be approximated by a power series (Lenzen 2000), considering all

373 elements up until a specific layer $k \in \mathbb{N}$. The truncation error estimate of the matrix layer

374 approach (TEE_MLA) for sector $u$ results as:

$375 T E E_{-} M L A_{u}=\frac{\left(f(I-A)^{-1}\right)_{u}-\left(f\left(\sum_{l=0}^{k} A^{l}\right) u\right.}{\left(f(I-A)^{-1}\right)_{u}} \quad \forall u \in M$.

376 In order to adjust the strictness of the cut-off criterion, the number of the maximal layer $k$ can 377 be varied.

$378<$ heading level 3> Exclusion of sectors using the matrix approach

379 To account for sectors frequently disregarded in PLCA analyses using the matrix approach we 380 define $S \subset M$ to be the set of sectors ignored in the analysis (Majeau-Bettez, Hawkins, and 381 Stromman 2011). Please refer to the Supporting Information (SI) for further details. $A$ is 382 modified such that entries referring to $S$ are set to zero, resulting in matrix $A^{*}$. The truncation 383 error estimates then result in:

$384 T E E_{i}=\frac{\left(f(I-A)^{-1}\right)_{u}-\left(f\left(I-A^{*}\right)^{-1}\right)_{u}}{\left(f(I-A)^{-1}\right)_{u}}$. (4) 
385 Although, literature indicates that specific capital goods are also ignored in process inventory 386 databases (Suh et al. 2004; Junnila 2006), we decline to implement a corresponding scenario, as 387 a precise list of sectors is missing. Hence, investigating their impact is left to future research.

$388<$ heading level 3> 10 - Path approach

389 The path approach, whose foundation has been described by Treloar (1997) and Norris (2002), 390 investigates the environmental impact of a process/product by tracing single flows in a typically 391 exponentially growing set of paths. This set of paths is often referred to as process tree in 392 literature, as upstream flows branch increasingly. Each element (single path), is described by the 393 equation $\omega_{o p \ldots q u}=A_{o}^{p} \cdot . \ldots \cdot A_{q}^{u} \cdot f^{u}$, which refers to associated $\mathrm{CO}_{2}$ emissions in sector $u$ of one 394 unit of output in sector $o$ with a corresponding production path $u \rightarrow q \rightarrow \cdots \rightarrow p \rightarrow$ $395 o, u, q, \ldots, p, o \in M$ (emissions have been released in sector $u$ ). We modify the approaches in 396 the literature to get a new procedure of tracing branches that is similar to tracing flows in PLCA. 397 Cut-off criteria are defined accordingly, that is flows which are likely to have an insignificant 398 contribution are deleted. Whether a flow with emissions $\omega_{o p \ldots q u}$ has a sufficiently significant 399 contribution to the study is judged on the basis of a threshold $t$. If $\omega_{o p \ldots q u}>t$ holds, all of $\omega_{o p \ldots q u}$ 400 first order upstream flows are added to the set of flows that need to be investigated. Otherwise 401 the flow is ignored, together with all of its upstream flows. This procedure is repeated until no 402 flows remain to be investigated.

$403<$ heading level 2> Defining scenarios

404 We use a modified IOLCA approach, see equation (4), to investigate how omitting service sectors 405 influences truncation error estimates. With regard to varying threshold rules, we recall that 
406 approaches in the literature estimating truncation errors consider different cut-off criteria. When

407 using the matrix layer approach, flows up to a specific layer are considered (Lenzen 2000). In the 408 case of a path approach, (ordered) flows have so far been cut off using an absolute threshold 409 (Treloar 1997) or a specific share of impacts within each layer (Norris 2002). To investigate the 410 influence of the choice of modeling frameworks, we first implement relative thresholds for the 411 path approach (each relative threshold corresponds to a specific absolute threshold by 412 transformation), where flows below a specific share of contribution are disregarded. Secondly we 413 use the matrix layer approach with varying maximal layer. A third path approach, using stochastic 414 thresholding, is implemented in the SI.

\section{$415<$ heading level $1>$ Results \& Discussion}

416 This section presents and discusses results of the scenarios introduced above. It then continues 417 with a discussion of the influence of various cut-off criteria and modeling frameworks on 418 truncation error estimates. Finally it provides implications for further research.

419 <heading level 2> Different cut-off criteria and modeling frameworks

420 In this subsection, different modeling frameworks are applied and compared, see figure 2 . The 421 matrix layer approach with varying maximal layers $k \in\{0,1,2,3\}$, and the process approach with 422 changing relative thresholds $t \in\{2 \%, 1 \%, 0.5 \%\}$ for each sector are implemented. Service 423 sectors are included as they would impact all modeling approaches equally and their exclusion 424 would not provide any additional relevant information. 

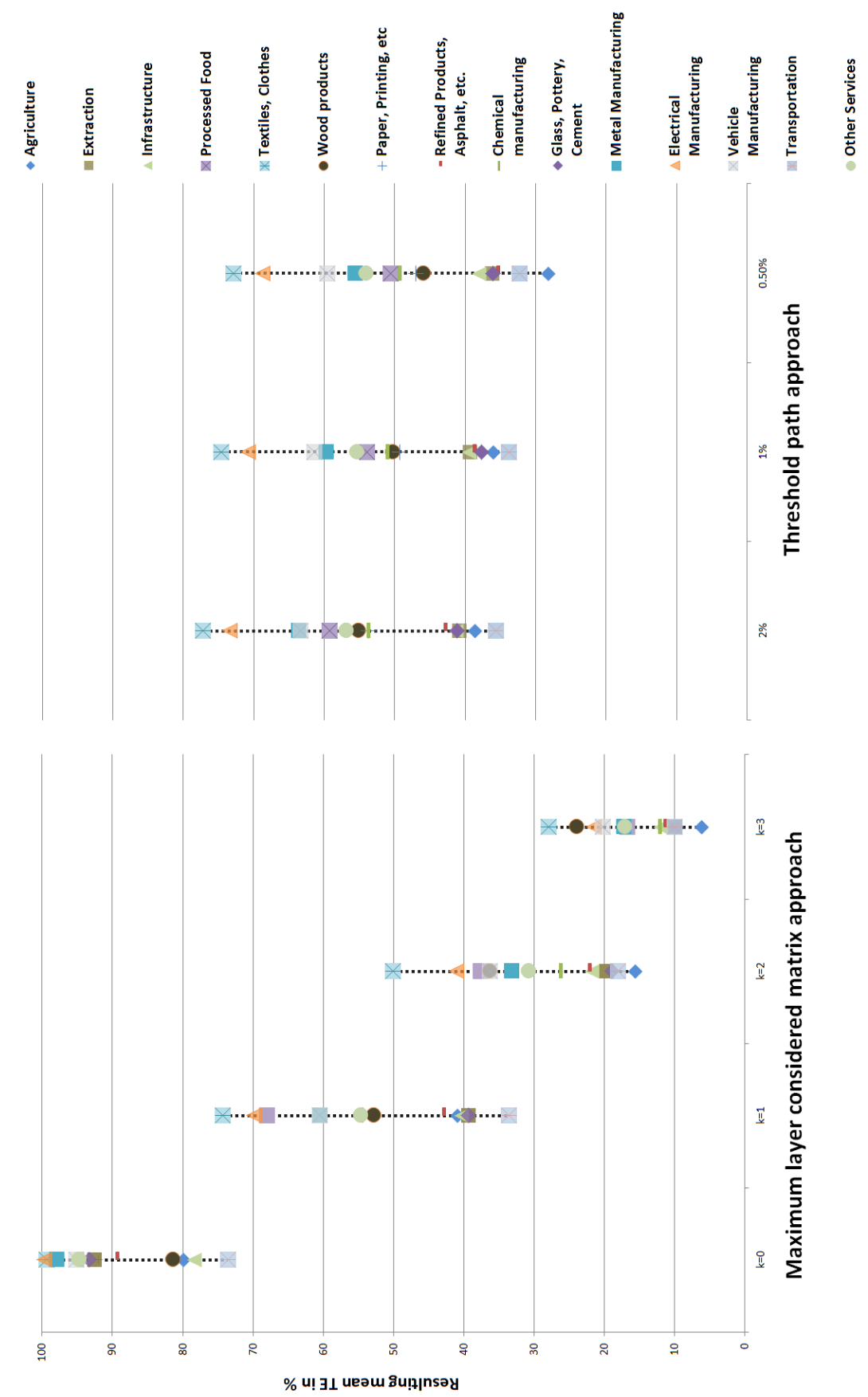

427 Figure 2: Mean truncation error estimate (for a sector group) when applying different cut-off criteria. Left: Matrix 428 layer approach with increasing maximum layers $(k \in\{0,1,2,3\})$. Right: Increasing strictness of (relative) threshold 429 ( $t \in\{2 \%, 1 \%, 0.5 \%\}$ ).

430

431 We find that for the path approach, mean truncation error estimates are between $28 \%$ and $76 \%$ 432 for different sector groups. Increasing the strictness of the threshold level from $2 \%$ to $0.5 \%$, leads 
433 to reductions in mean truncation error estimates of less than ten percentage points for all sector 434 groups. In comparison, results for the matrix layer approach show that significant reductions in 435 mean truncation error estimates for aggregated sectors occur with increasing strictness of cut436 off criteria. Mean truncation error estimates of $33 \%$ to $75 \%$ arise for all flows up to the first layer 437 (that is $k=1$ ). In contrast, truncation error estimates of $7 \%$ to $28 \%$ result when $k=3$. The 438 smallest truncation error estimations for both modeling frameworks (matrix layer and path 439 approach) are observable for agricultural and transportation sectors. The largest truncation 440 errors are identified for textile and electronic manufacturing sectors. These results are consistent 441 with the literature (Lenzen 2000). They reveal that the matrix layer approach is sensitive to an 442 increase in the strictness of the cut-off criterion, whereas the path approach is not. This is 443 because each approach incorporates flows differently. The matrix layer approach considers 444 whole flow layers, independent of the size of individual flows, whereas the path approach 445 considers single flows. The results become more distinct when investigating the distribution of 446 truncation error estimates (figure 3).

447 The distribution of truncation error estimates in the path approach, slowly shifts to the left when 448 the relative threshold is reduced (figure 3). In contrast, when increasing the strictness of cut-off 449 criteria for the matrix layer approach, the entire distribution quickly shifts to the left. 

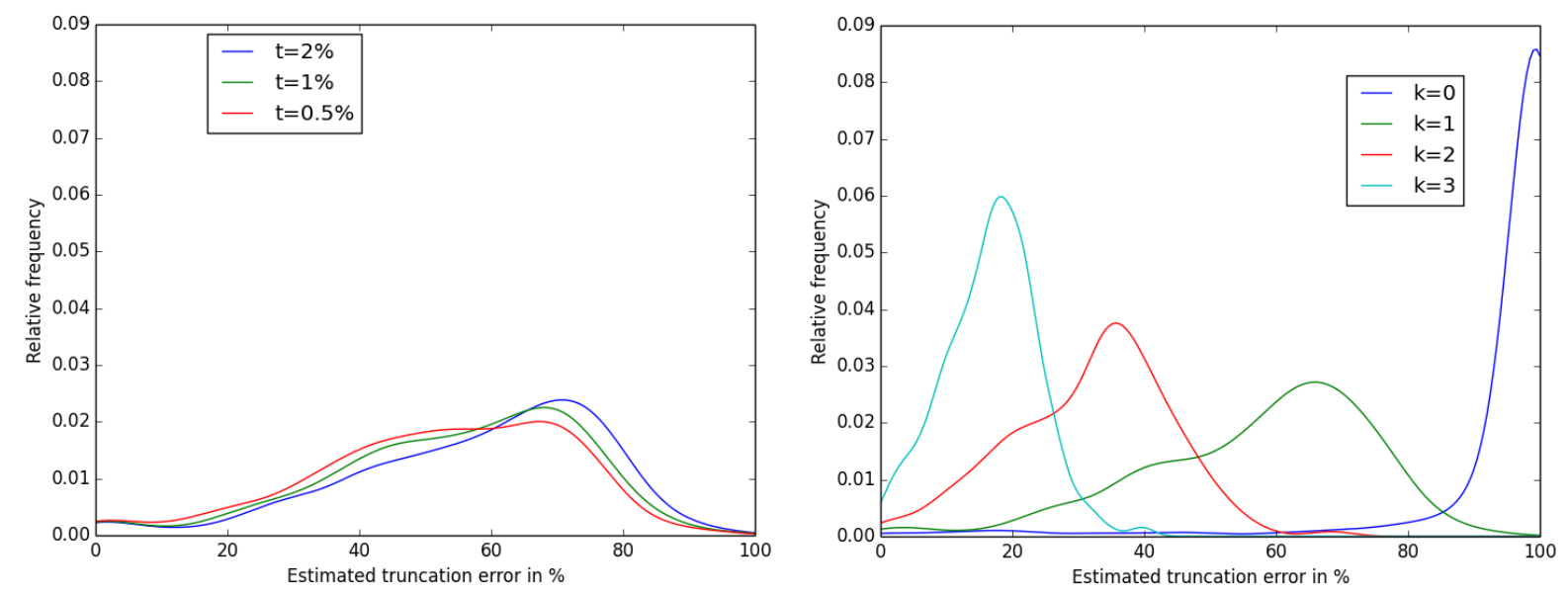

452

453

454 Figure 3: Relative distribution of truncation error estimates derived from the implemented path 455 approach (left) and relative distribution of truncation error estimates derived from the matrix layer 456 approach (right).

457

458 The results imply that there is a tremendous decline in the contribution of single flows within 459 higher flow layers. More importantly, the outcomes indicate that truncation errors of traditional 460 PLCA applications, which iteratively trace flows, are barely reduced when the strictness of the 461 cut-off criterion is increased. As a further reduction of the relative threshold imposes 462 computational difficulties, we cannot give a threshold $t$ that is sufficiently small to reduce 463 truncation errors below a specific level.

464 The implemented scenarios, using a single database, show that large differences in resulting 465 truncation error estimates can be observed. This depends on the cut-off criterion, underlying 466 framework, and strictness of thresholds chosen. Thus modeling specifications have a significant 467 influence on estimating the PLCA truncation error. 
$469<$ heading level 2> Disregarded sectors in PCLA

470 Our results show that ignoring service sectors not covered by life-cycle inventory databases, as

471 identified by Majeau-Bettez et al. (2011), causes median truncation error estimates of 3-13\%

472 depending on the sector group being analyzed; results vary across different sectors. The largest

473 truncation error estimates are located in manufacturing sectors (see figure 4), where median

474 truncation error estimates exceed $10 \%$. For a few specific sectors, such as fishing, electronic

475 computer manufacturing, computer storage, broadcast and wireless communications equipment

476 or analytical laboratory instruments, truncation error estimates can even exceed $20 \%$. The results

477 therefore indicate that disregarding specific sectors can be relevant. The smallest median 478 truncation error estimates occur in agricultural sectors, indicating that a high proportion of 479 emissions are associated with tier zero activities. As the most important direct emissions in the 480 agricultural sectors are greenhouse gases other than $\mathrm{CO}_{2}$ (Peters et al. 2010), analyzing different 481 impact categories could even augment the outcome by further reducing the estimate. 


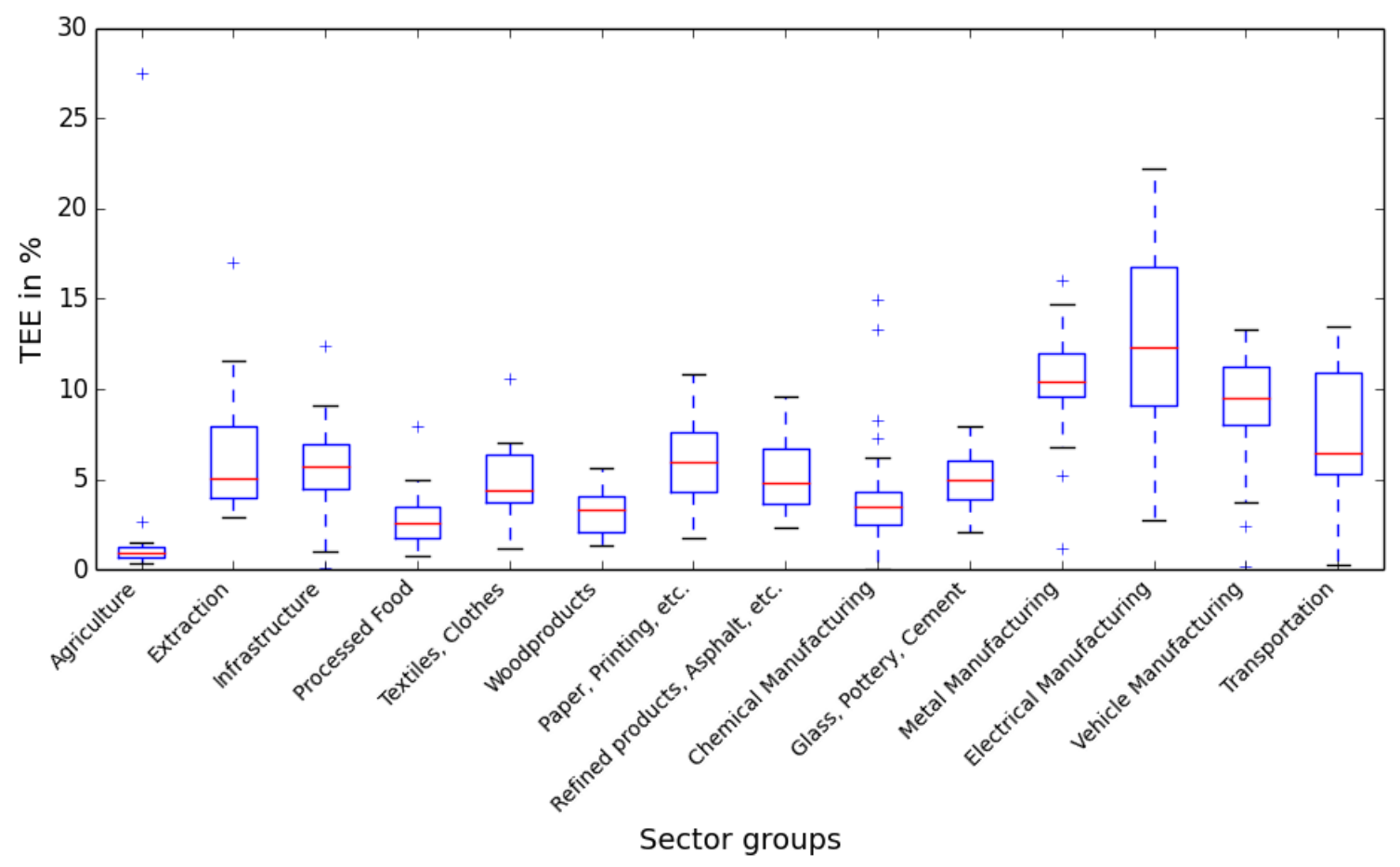

484 Figure 4: Influence on PLCA truncation error estimates through the disregard of service-related sectors that are 485 typically omitted in process databases. Red bars correspond to median truncation error estimates of the 486 corresponding sector group and blue boxes span from 1st to 3rd quartile. A detailed overview of aggregated sector 487 groups and disregarded service sectors can be found in the Supporting Information.

488

489

$490<$ heading level $1>$ Impact and future research

492 Conventional PLCA arguably suffers from truncation errors (Suh et al. 2004; Lenzen 2000). 493 Consequently total impacts assessed by PLCA (for example environmental impacts) associated 494 with specific products and processes remain unknown. Reviewing the literature on truncation 495 errors and their estimates, we find that the latter are influenced at three different levels: first 496 when estimating the impact itself, either through the PLCA or by approximating the PLCA 497 estimate; second when estimating the system complete counterfactual; and third when 
498 (possibly) concluding on two different systems, which is comparing IO or HLCA results with PLCA 499 results.

500

501 Our results show that large differences in estimates occur when investigating factors of these 502 different levels. Estimates crucially depend on the chosen modeling framework and the applied 503 cut-off criterion, even for a single database. Our results challenge explicit results and statements 504 on the size of PLCA truncation errors given in the literature, as the influence of the modeling 505 configurations has not been considered. In this respect the identified factors and the investigated 506 scenarios indicate that estimating PLCA truncation errors correctly is not possible; there are too 507 many interacting model factors at different levels that cannot simultaneously be accounted for. 508 Our results hence stress the necessity to carefully consider the influence of the modeling 509 framework on results in future assessments.

511 The findings are important for PLCA applicants in multiple dimensions. Firstly, they suggest that 512 not considering specific service sectors in process inventory databases can lead to relevant error513 prone results. Secondly, our results imply that the procedures for artificially curing the system 514 incompleteness of PLCA, for example by including an 10 correction term as in HLCA, cannot be 515 precisely evaluated as knowledge of the complete truncation error would be necessary.

516 Thirdly, the developed and implemented new variant of the path approach gives a (rough) 517 indication of the magnitude of PLCA truncation errors. We find that mean truncation error 518 estimates are likely to be in the range of $30 \%$ to $80 \%$. These depend on the sector group 
519 investigated. Nevertheless, we cannot quantify the influence on results that is introduced by our 520 modeling assumptions, for instance by using 10 data.

521 Fourthly, our results show that path approach truncation error estimations are relatively stable 522 across different threshold levels. This indicates that truncation errors associated with pure PLCA 523 can barely be reduced by increasing the strictness of the cut-off criterion. A feasible solution to 524 reduce truncation errors in a targeted manner might be the application of a preceding IOLCA 525 analysis (as an early-warning system) that orders the flows contributions as suggested by Treloar 526 (1997). It could indicate where relevant contribution shares are hidden in the process tree. HLCA 527 methodologies are widely judged to more precisely account for total (environmental) impacts. 528 The application of HLCA, such as the path exchange method (Lenzen and Crawford 2009), could 529 prevent severe truncation errors, while retaining the detail of PLCA.

530 Clearly our analysis alone could not consider all factors potentially influencing truncation errors 531 estimates that have been identified in the literature and in this article. It remains unclear how 532 they bias the results obtained. Hence, our results do not incorporate the full complexity necessary 533 to adequately quantify PLCA truncation errors, which seems to be difficult to achieve.

534 In the scenarios presented in the article we have evaluated the influence of some factors that are 535 characteristic for different PLCA truncation error estimates in the literature. Future investigations 536 need to assess the influence of other factors identified (for example differences in regional data 537 considered or network properties). More research is also required to clarify how the different 538 factors interact (for example, how do different network properties and varying cut-off criteria 539 jointly influence truncation error estimates). Finally, the most relevant factors biasing PLCA 
540 truncation error estimates have to be identified in order to help reduce unknown truncation 541 errors within PLCA applications.

\section{Acknowledgments}

543 We gratefully acknowledge the financial support of the German Research Association (DFG) and 544 the Collaborative Research Center 1026 "Sustainable Manufacturing - Shaping Global Value 545 Creation" of Technische Universität Berlin. The research leading to these results has also received 546 funding from the German Environmental Foundation (DBU) and the European Union Seventh 547 Framework Program FP7/2007-2013 under grant agreement no. 603864. The authors thank the 548 participants of the $24^{\text {th }}$ International Input-Output Conference in Seoul for their valuable 549 comments.

\section{References}

552 Alexeeva-Talebi, Victoria, Christoph Boehringer, Andreas Löschel, and Sebastian Voigt. 2012. "The Value553 Added of Sectoral Disaggregation: Implications on Competitive Consequences of Climate Change $554 \quad$ Policies." Energy Economics 34 (2): 127-42.

555 Applied Sustainability Center, University of Arkansas. Sylvatica. 2010. "Open IO." http://www.open$556 \quad$ io.org/.

557 British Standard Institute. 2011. "PAS 2050 - Specification for the Assessment of the Life Cycle

558 Greenhouse Gas Emissions of Goods and Services."

559 http://shop.bsigroup.com/upload/shop/download/pas/pas2050.pdf.

560 British Standards - ISO 14040. 2006. "Environmental Management - Life Cycle Assessment -

561 Requirements and Guidelines." British Standards.

562 British Standards - ISO 14044. 2006. "Environmental Management - Life Cycle Assessment -

563 Requirements and Guidelines." British Standards.

564 Bruckner, Martin, Günther Fischer, Sylvia Tramberend, and Stefan Giljum. 2015. “Measuring

565 Telecouplings in the Global Land System: A Review and Comparative Evaluation of Land

566 Footprint Accounting Methods." Ecological Economics 114: 11-21.

567 Crawford, Robert H., and André Stephan. 2013. "The Significance of Embodied Energy in Certified Passive 568 Houses." International Journal of Civil, Environmental, Structural, Construction and Architectural $569 \quad$ Engineering 7 (6): 427-33.

570 Finnveden, Göran, Michael Z. Hauschild, Tomas Ekvall, Jeroen B. Guinée, Reinout Heijungs, Stefanie $571 \quad$ Hellweg, Annette Koehler, David Pennington, and Sangwon Suh. 2009. "Recent Developments in $572 \quad$ Life Cycle Assessment." Journal of Environmental Management 91: 1-21.

573 Guinée, Jeroen B. 2011. "Life Cycle Assessment: Past, Present, and Future." Environmental Science \& $574 \quad$ Technology 45 (1): 90-96. 
575 Huang, Y. Anny, Manfred Lenzen, Christopher L Weber, Joy Murray, and H. Scott Matthews. 2009. "THE 576 ROLE OF INPUT-OUTPUT ANALYSIS FOR THE SCREENING OF CORPORATE CARBON FOOTPRINTS." 577 Economic Systems Research 21 (3): 217-42. doi:10.1080/09535310903541348.

578 IELab. 2017. “Industrial Ecology Virtual Laboratory." Accessed April 12. https://ielab.info/. 579 Joshi, Satish. 1999. "Product Environmental Life-Cycle Assessment Using Input-Output Techniques." $580 \quad J$ Journal of Industrial Ecology 3 (2-3): 95-120. doi:10.1162/108819899569449.

581 Junnila, Seppo I. 2006. "Empirical Comparison of Process and Economic Input-Output Life Cycle

582 Assessment in Service Industries." Environmental Science \& Technology 40: 7070-76.

583 Laurienti, Paul, J, Karen E Joyce, Qawi K Telesford, Jonathan H Burdette, and Satoru Hayasaka. 2011.

$584 \quad$ "Universal Fractal Scaling of Self-Organized Networks." Physica A 390: 3608-13.

585 Lee, Chia-Ho, and Hwong-Wen Ma. 2013. "Improving the Integrated Hybrid LCA in the Upstream Scope 3

586 Emissions Inventory Analysis." International Journal of Life Cycle Assessment 18: 17-23.

587 Lenzen, Manfred. 2000. "Errors in Conventional and Input-Output-based Life-Cycle Inventories."

$588 \quad J$ Journal of Industrial Ecology 4 (4): 127-48.

589 Lenzen, Manfred, and Robert Crawford. 2009. "The Path Exchange Method for Hybrid LCA."

$590 \quad$ Environmental Science \& Technology 43: 8251-56.

591 Lenzen, Manfred, and Christopher Dey. 2000. "Truncation Error in Embodied Energy Analyses of Basic

$592 \quad$ Iron and Steel Products." Energy 25: 577-85.

593 Lenzen, Manfred, Daniel Moran, Anik Bhaduri, Keiichiro Kanemoto, Maksud Bekchanov, Arne Geschke,

594 and Barney Foran. 2013. "International Trade of Scarce Water." Ecological Economics 94: 78-85.

595 Lenzen, Manfred, and Graham Treloar. 2002. "Embodied Energy in Buildings: Wood versus

596 concreteFreply to B. Orjesson and Gustavsson." Energy Policy 30: 249-55.

597 - - . 2003. "Differential Convergence of Life-Cycle Inventories toward Upstream Production Layers."

$598 \quad J o u r n a l$ of Industrial Ecology 6 (3-4): 137-60.

599 Majeau-Bettez, Guillaume, Troy R. Hawkins, and Anders Hammer Stromman. 2011. "Evaluation of

600 Process- and Input-...: Output-Based Life Cycle Inventory Data with Regard to Truncation and

601 Aggregation Issues." Environmental Science \& Technology 45: 10170-77.

602 Mongelli, Ignazio, Sangwon Suh, and Gjalt Huppes. 2005. "A Structure Comparison of Two Approaches to

603 LCA Inventory Data, Based on the MIET and ETH Databases." International Journal of Life Cycle

$604 \quad$ Assessment 10 (5): 317-24.

605 Norris, Gregory A. 2002. "Life Cycle Emission Distributions Within the Economy: Implications for Life

$606 \quad$ Cycle Impact Assessment." Risk Analysis 22 (5): 919-30.

607 PE International. 2015. "GaBi Software." http://www.gabi-software.com/deutsch/index/.

608 Peters, Gregory M., Hazel V. Rowley, Stephen Wiedemann, Robyn Tucker, Michael D. Short, and

$609 \quad$ Matthias Schulz. 2010. "Red Meat Production in Australia: Life Cycle Assessment and

$610 \quad$ Comparison with Overseas Studies." Environmental Science \& Technology 44: 1327-32.

611 Rowley, Hazel V., Sven Lundie, and Gregory M. Peters. 2009. "A Hybrid Life Cycle Assessment Model for

612 Comparison with Conventional Methodologies in Australia." The International Journal of Life

$613 \quad$ Cycle Assessment 14: 508-16.

614 Simas, Moana S., Laura Golsteijn, Mark A. Huijbregts, Richard Wood, and Edgar G. Hertwich. 2014. "The

615 'Bad Labor' Footprint: Quantifying the Social Impacts of Globalization." Sustainability 6 (11):

616 7514-40.

617 Steen-Olsen, Kjartan, Anne Owen, Edgar G. Hertwich, and Manfred Lenzen. 2015. "Effects of Sector

618 Aggregation on CO2 Multipliers in Multiregional Input-output Analyses." Economic Systems 619 Research 26 (3): 284-302.

620 Su, Bin, H. C. Huang, B. W. Ang, and P. Zhou. 2010. "Input-output Analysis of CO2 Emissions Embodied in

621 Trade: The Effects of Sector Aggregation." Energy Economics 32: 166-75.

622 Suh, Sangwon, and Gjalt Huppes. 2005. "Methods for Life Cycle Inventory of a Product." Journal of

$623 \quad$ Cleaner Production 13: 687-97. 
624 Suh, Sangwon, Manfred Lenzen, G. Treloar, H. Hondo, Arpad Horvath, G. Huppes, O. Jolliet, et al. 2004.

$625 \quad$ "System Boundary Selection for Life Cycle Inventories Using Hybrid Approaches." Environmental

$626 \quad$ Science \& Technology 38 (3): 657-64.

627 Treloar, Graham. 1997. "Extracting Embodied Energy Paths from Input-Output Tables: Towards an

628 Input-Output-Based Hybrid Energy Analysis Method." Economic Systems Research 9 (4): 375-88.

629 Tukker, Arnold, and Erik Dietzenbacher. 2013. "Global Multiregional Input-Output Frameworks: An

$630 \quad$ Introduction and Outlook." Economic Systems Research 25 (1): 1-19.

631 doi:10.1080/09535314.2012.761179.

632 Ward, Hauke, Mia Burger, Ya-Ju Chang, Paul Fürstmann, Sabrina Neugebauer, Alexander Radebach,

$633 \quad$ Gunther Sproesser, et al. 2016. "Assessing Carbon Dioxide Emission Reduction Potentials of

$634 \quad$ Improved Manufacturing Processes Using Multiregional Input Output Frameworks." Journal of

$635 \quad$ Cleaner Production.

636 Wiedmann, Thomas O., Heinz Schandl, Manfred Lenzen, Daniel Moran, Sangwon Suh, James West, and

637 Keiichiro Kanemoto. 2013. "The Material Footprint of Nations." Proceedings of the National

$638 \quad$ Academy of Sciences. doi:doi:10.1073/pnas.1220362110.

639 Wiedmann, Thomas O., Sangwon Suh, Kuishuang Feng, Manfred Lenzen, Adolf Acquaye, Kate Scott, and

$640 \quad J o h n$ R. Barrett. 2011. "Application of Hybrid Life Cycle Approaches to Emerging Energy

641 Technologies - The Case of Wind Power in the UK." Environmental Science \& Technology 45:

$642 \quad 5900-5907$.

643 Zhang, Yi, Erin L. Gibbemeyer, and Bhavik R. Bakshi. 2014. "Empirical Comparison of Input-Output

$644 \quad$ Methods for Life Cycle Assessment." Journal of Industrial Ecology 18 (5): 734-46.

645

646

647

\section{About the authors}

649 Hauke Ward is a PhD candidate at the Technische Universität Berlin in Berlin, Germany. He is also 650 affiliated with the Mercator Research Institute on Global Commons and Climate Change, Berlin, 651 Germany and the Potsdam Institute for Climate Impact Research, Potsdam, Germany. Leonie 652 Wenz is a researcher at the Potsdam Institute for Climate Impact Research, Potsdam, Germany. 653 She is affiliated with the Mercator Research Institute on Global Commons and Climate Change, 654 Berlin, Germany and the Institute of Physics at the University of Potsdam, Potsdam, Germany. Jan 655 C Steckel is a researcher at the Mercator Research Institute on Global Commons and Climate 656 Change, Berlin, Germany, where he heads the group "Climate and Development". He is also 657 affiliated with the Technische Universität Berlin in Berlin, Germany and the Potsdam Institute for 658 Climate Impact Research, Potsdam, Germany. Jan C Minx is a professor at the Hertie School of 659 Governance, Berlin, Germany. He is also affiliated with the Mercator Research Institute on Global 660 Commons and Climate Change, Berlin, Germany, where he heads the group "Applied 661 Sustainability Science".

\footnotetext{
i i Please note that we chose a database representing the US economy in 2002. Although, these data are non-actual, the derived results are valid and representative for modeling approaches. This database was been chosen because it coincides with the one used by Majeu-Bettez et al. (2011).
} 
ii In the latest version of (British Standards - ISO 14044 2006) it is stated that "The initial system boundary shall be revised, as appropriate, in accordance with the cut-off criteria established in the definition of the scope." 